\title{
Mathematisches Modell für die Brammenerwärmung im Hubbalkenofen
}

\author{
Werner Pollhammer ${ }^{1,2}$, Christoph Spijker², Jakob Six ${ }^{3}$, Daniel Zoglauer ${ }^{3}$ und Harald Raupenstrauch ${ }^{2}$ \\ ${ }^{1} \mathrm{~K} 1-\mathrm{MET} \mathrm{GmbH}$, Linz, Österreich \\ ${ }^{2}$ Lehrstuhl für Thermoprozesstechnik, Montanuniversität Leoben, Leoben, Österreich \\ ${ }^{3}$ voestalpine Stahl GmbH, Linz, Österreich
}

Eingegangen 20. Juni 2018; angenommen 6. August 2018; online publiziert 4. September 2018

\begin{abstract}
Zusammenfassung: In der Stahlindustrie wird der Hubbalkenofen dazu verwendet, die Brammen vor dem Walzen zu erhitzen. Um die hohen Qualitätsanforderungen zu erreichen, wird ein sehr gleichmäßiges Temperaturprofil in den Brammen verlangt. Mithilfe von Computational Fluid Dynamics (CFD) wurde in ANSYS Fluent 15 ein Modell zur Berechnung und Analyse der Wärmeströme in einem Hubbalkenofen erstellt. Das Modell beinhaltet die Modellierung der Verbrennung, Strahlung, Turbulenz sowie Brammenbewegung und Zunderaufbau an der Brammenoberfläche. Die Ergebnisse aus der Simulation und einem Schleppversuch wurden anschließend verglichen, und es zeigt sich, dass das Modell in der Lage ist, die Wärmeströme im Ofen richtig abzubilden. In weiterer Folge wurde das entwickelte Modell dazu verwendet, die Ursachen von Temperaturinhomogenitäten in den Brammen festzustellen. Mittels einer Reihe von Simulationen konnte anschließend ein Ofenprogramm gefunden werden, welches durch eine Leistungsverschiebung der Brenner eine deutliche Reduktion der Temperaturunterschiede in den Brammen ermöglicht.
\end{abstract}

Schlüsselwörter: Hubbalkenofen, CFD-Modell, Simulation, Brammenerwärmung

\section{Mathematical Model of a Walking Beam Furnace}

Abstract: In the steel industry, the walking beam furnace is used to heat up and reheat steel slabs for the rolling process. In order to meet the demands for high quality steel, a very homogeneous temperature profile in the slabs is required. Using Computational Fluid Dynamics (CFD) in ANSYS Fluent 15, a walking beam furnace has been investigated to analyse the causes of inhomogeneous temper-

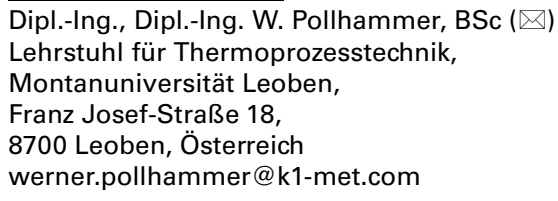

ature distribution in the steel slabs. The model includes the description of combustion, radiation turbulence as well as slab movement and scale formation on the steel. The results out of a simulation and a towing test have been compared and it has turned out that the model is able to predict the heat fluxes in the furnace very precisely. Next the model has been used to find the reasons of inhomogeneous temperature distribution in the steel slabs. With a certain number of simulations, a furnace program has been found which reduces the temperature differences in the steel slabs just by adjusting the power of the burners.

Keywords: Walking beam furnace, CFD, Model, Simulation, Steel slabs heating

\section{Einleitung}

Für die Herstellung von hochqualitativen Stahlblechen ist eine homogene Temperaturverteilung in den Brammen während des Walzprozesses von hoher Bedeutung. Um diese Homogenität zu gewährleisten bzw. überhaupt zu ermöglichen, bedarf es einer sehr genauen Kenntnis der Wärmeströme im vorgeschalteten Prozessaggregat, dem Hubbalkenofen. Im Rahmen dieser Arbeit wurde eine numerische Strömungssimulation (CFD-Modell) für einen Ofen dieser Art erstellt. Das dabei entwickelte Modell berücksichtigt neben der Balkenbewegung unter anderem die Strahlungseinflüsse und Wärmeverluste an den Ofenwänden sowie die Isolationswirkung der Balkenverzunderung. Aufgrund der hohen Temperaturen im Ofenraum sind exakte Temperaturmessungen der Ofenatmosphäre sehr schwierig und wenn dann nur punktuell und mit sehr hohem Aufwand durchführbar. Mithilfe des entwickelten mathematischen Modells wird es nun möglich, die Wärmeströme im gesamten Ofenaggregat zu berechnen und Fallstudien durchzuführen, ohne dabei den laufenden Betrieb am Ofen zu beeinträchtigen. 


\section{Modellbeschreibung}

\subsection{Ofengeometrie}

Der im Modell abgebildete Hubbalkenofen besteht aus 48 Brennern, welche mit Gichtgas und Erdgas betrieben werden. Da der Ofen symmetrisch betrieben wird, das heißt, dass gegenüberliegende Brenner die gleiche Leistung aufweisen, wurde nur eine Ofenhälfte simuliert. Dies reduziert die Zellenanzahl des Gesamtmodells von 440 Mio. Tetraedern auf 220 Mio.. Um auszuschließen, dass sich gegenüberliegende Brenner gegenseitig beeinflussen, wurde vor der Geometrieerstellung ein Testfall erstellt, welcher die gegenseitige Strömungsablenkung zweier gegenüberliegender Brenner darstellt. Dabei konnte herausgefunden werden, dass es aufgrund des großen Abstands von $12 \mathrm{Me}-$ tern zu keiner gegenseitigen Brennerbeeinflussung kommt und die Verwendung einer Symmetrieebene somit möglich und legitim ist.

\subsection{Wärmewiderstandsmodell der Wand}

Der Aufbau der Ofenwand ist durchaus komplex. Diese besteht aus mehreren Schichten verschiedener Feuerfestwerkstoffe, welche selbst zum Teil eine hohe Abhängigkeit der Wärmeleitfähigkeit von der Temperatur besitzen. Die Ofenwand steht dabei auch im Strahlungsaustausch mit der Ofenraumatmosphäre und spielt eine große Rolle bei der Wärmeeinbringung in die Brammen. Um eine präzise Temperaturvorhersage zu ermöglichen, müssen diese Aspekte berücksichtigt werden. Dazu wurde ein Wärmeleitmodell der Ofenwand entwickelt, welches eine effiziente Implementierung findet. Mithilfe von Matlab wurde die Ofenwand, wie in Abb. 1 dargestellt, eindimensional diskreditiert.

Die Auflösung erfolgte dabei in 100 Zellen, und die Wärmeleitungsgleichung wurde mittels finiter Differenzen in Matlab iterativ gelöst. Dabei wurde die temperaturabhängige Wärmeleitfähigkeit in jeder Zelle berücksichtigt. Als konstante Randbedingungen für die Berechnung wurde neben der Vorgabe des Schichtaufbaus die Oberflächentemperatur an der Umgebung gesetzt. Anschließend wurde die Temperatur der Ofenraumatmosphäre schrittweise erhöht

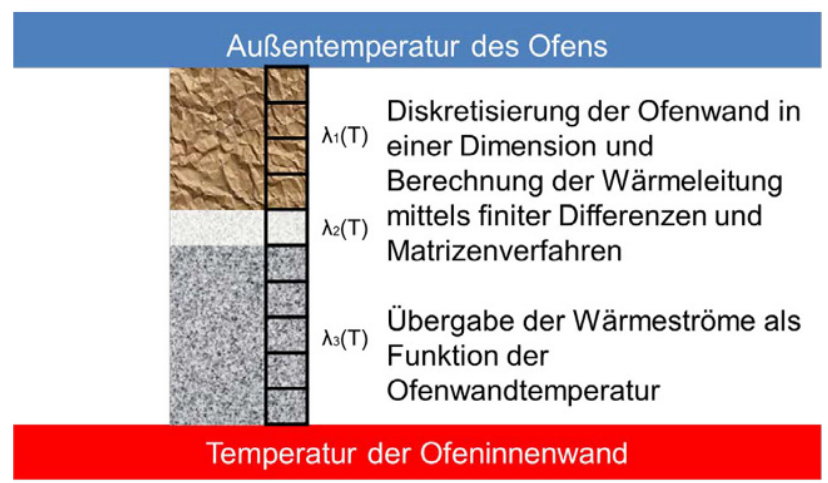

Abb. 1: Thermisches Ofenwandmodell und der Wärmestrom durch den Wandaufbau berechnet. Aus diesen Daten kann ein Polynom generiert werden, welches den Wärmestrom durch die Ofenwand in Abhängigkeit der Ofenwandtemperatur im Innenraum unter Berücksichtigung des Wandaufbaus und der temperaturabhängigen Wärmeleitfähigkeiten der verwendeten Feuerfestmaterialien stationär beschreibt. Mithilfe einer UDF (User Defined Function) wird dieser Wärmestrom an jeder Ofenwandzelle der Simulation berechnet und in der Energiebilanz berücksichtigt.

\subsection{Ermittlung der Emissionskoeffizienten der Feuerfestmaterialien und der Stahlbramme}

Wie sich in den Ergebnissen zeigt, werden ca. $92 \%$ der in die Bramme eingebrachten Energie in Form von Wärmestrahlung übertragen und lediglich $8 \%$ durch Konvektion. Daher ist die exakte Kenntnis der Emissionskoeffizienten der verwendeten Feuerfestmaterialien sowie der Stahlbramme von besonderer Bedeutung. Im Rahmen dieser Arbeit wurden die Werte im Labor bestimmt. Dabei wurde die Probe mit einer Bohrung versehen und ein Thermoelement in der Probenmitte und eines im Ofenraum platziert. Durch ein kleines Loch im Ofen wurde mittels Wärmebildkamera ein Thermogramm aufgenommen, welches anhand der Temperaturen der Thermoelemente einen Rückschluss auf den Emissionskoeffizienten ermöglicht. Die Messung wurde dabei in Schritten von 300 bis $1100^{\circ} \mathrm{C}$ in $200^{\circ} \mathrm{C}$ Schritten durchgeführt. Nach Änderung der Temperatur am Ofen wurde sichergestellt, dass die Temperatur der Probenmitte und der Ofenraumtemperatur angeglichen waren. Nur so kann davon ausgegangen werden, dass die Oberflächentemperatur der Probe auch der Kerntemperatur entspricht, kein Wärmfluss mehr über die Oberfläche stattfindet und ein stationärer Zustand vorliegt. Es zeigt sich dabei ein leicht steigender Trend der Emissionskoeffizienten aller vermessenen Proben von 0,90 bei $300^{\circ} \mathrm{C}$ auf 0,95 bei $1100^{\circ} \mathrm{C}$.

\subsection{Brammenbewegung}

Um die Brammenbewegung im Modell zu berücksichtigen, bedient man sich eines sogenannten MRF (Multi Reference Frame). Dieses addiert im Bereich der Brammenbewegung zum statischen Netz einen konvektiven Anteil hinzu, welcher die Bewegung der Brammen abbildet. Im Gegensatz zu einem dynamischen Netz kann man so Probleme durch die andauernde Neuvernetzung und numerischen Instabilitäten bei gleichbleibender Genauigkeit vermeiden.

\subsection{Turbulenzmodellierung}

Für die Modellierung der Gasphasenkinetik ist die Beschreibung der Turbulenz sehr wichtig. In diesem Falle wurde das

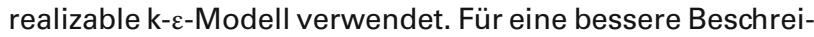
bung der Flammenbeschleunigung wurde, wie in der Literatur vorgeschlagen, die Konstante $C_{2 \varepsilon}$ von 1,9 auf 1,8 reduziert [1, 2]. Im Modell wurde dabei eine isotropische turbu- 
lente Viskosität verwendet. Somit besteht das Turbulenzmodell aus zwei Transportgleichungen, der k-Gleichung für die turbulente kinetische Energie der Wirbel in der Strömung und der $\varepsilon$-Gleichung für die Dissipation von $\mathrm{k}$.

\subsection{Verbrennungsmodellierung}

Für die Beschreibung der Verbrennung in einer Modellgröße dieses Ofens ist das Flameletmodel die effizienteste Lösung [3]. Dabei wird die gesamte Verbrennungschemie vor der eigentlichen Simulation gelöst und in Tabellen abgelegt. Dazu wird eine eindimensionale Gegenstromdiffusionsflamme mit Hilfe eines detailchemischen Modells gelöst und die sogenannten Flamelets generiert. Anschließend erfolgt die PDF (Probability Density Function) - Integration um die Turbulenzfluktuationen mit zu berücksichtigen. Diese dabei generierten Daten werden in der PDF-Tabelle abgelegt, welche die tabellierten Verbrennungswerte enthält. Zu diesen Daten gehören unter anderem Reaktionsraten, Verbrennungswärme und Speziesinformationen. Anhand der molekularen Zusammensetzung der Gasphase, der Temperatur und Turbulenz werden die Stoffumsätze und Wärmetönungen aus der PDF-Tabelle für jede Zelle ausgelesen. Im Gegensatz zu einer kontinuierlichen Berechnung der Detailchemie bringt dies eine Berechnungsbeschleunigung um den Faktor 100.

\subsection{Strahlungsmodellierung}

Zur Beschreibung des Wärmestroms durch Strahlung wurde das Discrete Ordinate Model (DOM) in Kombination mit dem Weighted Sum of Grey Gases Model (WSGGM) verwendet [4,5]. Diese beiden Modelle ermöglichen in Kombination eine sehr genaue Beschreibung der Strahlung. Das DOM bildet dabei von jeder Randzelle eine bestimmte Anzahl von Strahlen in den hemisphären Raum und beschreibt den Strahlungstransport in der Geometrie unter Berücksichtigung von Abschattungseffekten. Das WSGGM bildet dabei einen von der Gasmischung abhängigen Emissionsund Absorptionskoeffizienten, welcher für den Strahlungstransport benötigt wird.

\subsection{Verzunderungsmodell}

Die Verzunderung der Brammen spielt für den Wärmetransport eine sehr große Rolle. Durch die Zunderbildung an der Brammenoberfläche bildet sich eine Schicht mit schlechter Wärmeleitfähigkeit aus. Diese isoliert zunehmend die Bramme und senkt den Wärmeeintrag in den Stahl. Die Zunderbildung wird dabei mittels eines Modells aus der Literatur beschrieben [6]. Das Modell beschreibt den zeitlichen Aufbau des Zunders an der Bramme und berücksichtigt dabei den Transportwiderstand von Eisen durch die Zunderschicht. Für das Modell werden die Sauerstoffkonzentration an der Stahloberfläche, die Temperatur des Stahls sowie die Stoffdaten der Gasphase und des Stahls benötigt. Der Zunderaufbau wird durch ein stationäres 1D
Modell mittels finiten Differenzen berechnet. Dabei stellt die Zunderschichtdicke die einzige Transportvariable dar, welche mit der Brammengeschwindigkeit transportiert wird. Die Stoffübergangskoeffizienten und die Sauerstoffkonzentration an der Brammenoberfläche werden aus dem CFDModell ausgelesen. Die Temperatur der Brammen wird entweder aus den Messdaten für den Schleppversuch verwendet oder iterativ mit den Simulationsdaten ermittelt, für den Fall dass keine Brammentemperatur zur Verfügung steht. Die Daten werden über die Brammenbreite gemittelt und in richtig der Brammenbewegung diskreditiert. So entsteht das in Vorschubrichtung eindimensional diskretisierte Modell. An jedem Knotenpunkt wird die Reaktionsrate für den Zunderaufbau berechnet und zur Transportvariable Zunderdicke addiert. Die Zunderdicke wird durch den Ofen transportiert und liefert als Ergebnis die von der Ofenkoordinate abhängige Zunderschichtdicke auf der Bramme. Bei der Berechnung der Zunderschichtdicke wird einerseits die Diffusionskontrolle von Eisen durch die Zunderschicht und andererseits die Diffusionskontrolle von Sauerstoff an die Oberfläche berücksichtigt. Die Diffusion von Eisen im Zunder wird durch GI. 1 aus der Literatur bestimmt [6]

$$
D(T)=1,21 e^{-\frac{1,24 E 5}{R T}}
$$

Der Zuwachs des Zunders wird mittels stationären Diffusionsansatzes anhand von Gl. 2 berechnet. Um eine Division durch null zu vermeiden, wird am Ofeneintrag eine vernachlässigbare Zunderdicke von $1 \mu \mathrm{m}$ als Startwert vorgegeben.

$$
\frac{d s}{d t}=\frac{D(T)}{s+s m a l l} \frac{M M_{\text {Zunder }}}{\rho_{\text {Zunder }}}
$$

Aber nicht der gesamte Zunder verbleibt auf der Bramme. So ist im Ofenbetrieb zu sehen, dass es in den letzten $20 \%$ der Ofenlänge zum Abplatzen des Zunders kommt und dieser dann am Ofenboden zu liegen kommt. Deshalb wird der Zunderaufbau ab dieser Ofenposition gestoppt, und es wird davon ausgegangen, dass der neu entstehende Zunder von der Bramme abplatzt.

Aus diesen Daten wird anschließend ein Polynom gebildet, welches den Wärmewiderstand der Zunderschicht in Abhängigkeit der Ofenkoordinate beschreibt. Dabei wird neben der Zunderschichtdicke auch die temperaturabhängige Wärmeleitfähigkeit des Zunders berücksichtigt.

\section{Modellvalidierung}

Um das Modell zu validieren, wurde ein Vergleich mit einem Schleppversuch durchgeführt. Dazu wurde eine Messbramme, welche mit Thermoelementen versehen ist, durch den Ofen transportiert und das Temperaturprofil der Bramme und der Gasatmosphäre aufgezeichnet. Über die Vorschubgeschwindigkeit und der zeitlichen Temperaturaufzeichnung kann so ein örtlich aufgelöstes Temperaturprofil erstellt werden. Abb. 2 zeigt diesen Vergleich zwischen der Simulation und dem Schleppversuch. Es zeigt sich, dass eine genaue Vorhersage des Temperaturprofils mittels Si- 


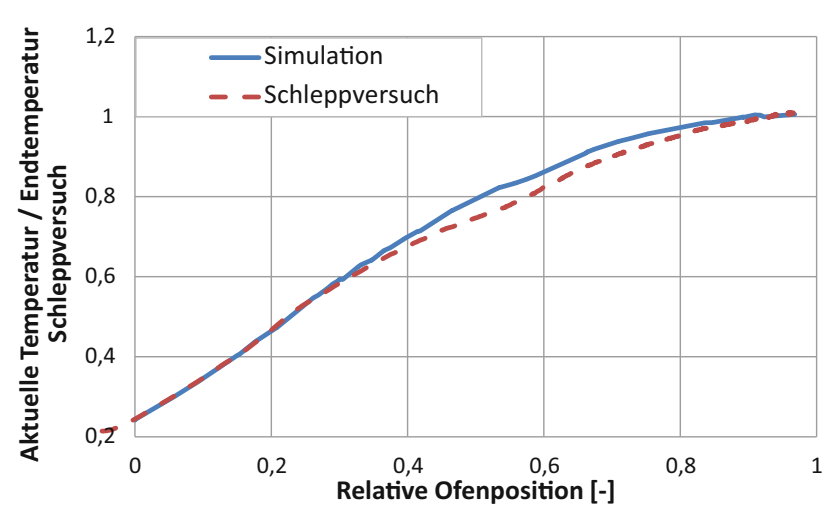

Abb. 2: Vergleich der Brammentemperatur aus Simulation und Schleppversuch

mulation möglich ist und das Modell für weitere Fallstudien verwendet werden kann.

\section{Ergebnisse}

Nach der erfolgreichen Modellvalidierung wurde das Modell dazu verwendet, die Ursachen für Inhomogenitäten in der Brammentemperaturverteilung zu studieren. Es zeigt sich im Betrieb, dass vor allem bei hohem Brammendurchsatz und hoher Ofenleistung ein durchhängendes Temperaturprofil entsteht. Das heißt, dass der Stahl in der Ofenmitte kühler als am Rand ist. Dieses Verhalten sollte vermieden werden, daher wurde es mittels CFD-Modell genau untersucht. Dabei stellte sich heraus, dass die Ursache für die Temperaturunterschiede in der Fahrweise und den Strömungsprofilen der Brenner liegt und dass die Wärmestrahlung für die Überhitzung am Rand verantwortlich ist. Abb. 3 zeigt dieses unerwünschte Brennerverhalten sehr gut. In der unteren Brennerebene wird bis zur Brammenmitte (rechter Bildrand) ein annähend gleichmäßiger Wärmeeintrag ermöglicht, da die Strahlungsintensität unterhalb der Bramme über die gesamte Breite annähernd gleichmä-
Big verteilt ist. An der Oberseite der Bramme zeigt sich ein anderes Verhalten, welches für die höhere Temperatur am Brammenrand (links) verantwortlich ist. Zwischen dem ersten und zweiten Balken bildet sich vor dem Brenner ein Hotspot mit einer hohen Wärmestrahlungsdichte. Diese Wärme wird unmittelbar am Brammenrand übertragen und heizt diese dort stärker auf als in den Zonen mit geringerer Strahlungsdichte weiter rechts.

Im Rahmen der Studie wurden verschiedene Brennerprogramme getestet und die Auswirkungen auf die Brammenerwärmung untersucht. So konnte letztlich durch eine Lastverschiebung und einer sehr genaue Leistungsanpassung der Brenner ein Ofenprogramm gefunden werden, welches diese Hotspotbildung vermeidet und ein homogeneres Temperaturprofil ermöglicht. Wichtig dabei anzumerken ist, dass die Brenner keinesfalls einzeln betrachtet werden dürfen. Es zeigt sich in den Analysen, dass sich benachbarte Brenner durchaus beeinflussen und Änderungen in einer Brennerebene auch zu Änderungen in anderen Brennerebenen führen. Deshalb ist eine gesamtheitliche Betrachtung des Ofens unbedingt notwendig, um präzise Aussagen treffen zu können.

\section{Zusammenfassung}

Um die Ursache für Inhomogenitäten in der Temperaturverteilung von Brammen zu studieren, wurde ein detailliertes Ofenmodell für den Hubbalkenofen erstellt. In diesem CFD-Model sind eine detaillierte Beschreibung von Turbulenz, Verbrennung, Strahlung und Brammenbewegung sowie Verzunderung implementiert. Es zeigte sich in der Modellentwicklung, dass nur mittels Berücksichtigung all dieser Faktoren eine präzise Temperaturvorhersage ermöglicht wird. Nach der erfolgreichen Modellvalidierung anhand eines Schleppversuchs wurde das Modell dazu verwendet, verschiedene Leistungseinstellungen an den Brennern und dessen Auswirkungen auf die Brammenerwärmung zu studieren. Es zeigte sich dabei, dass durch eine Hotspotbildung in den Brennern örtlich sehr unterschied-
Abb. 3: Strahlungswärmestrom in Brennerebene

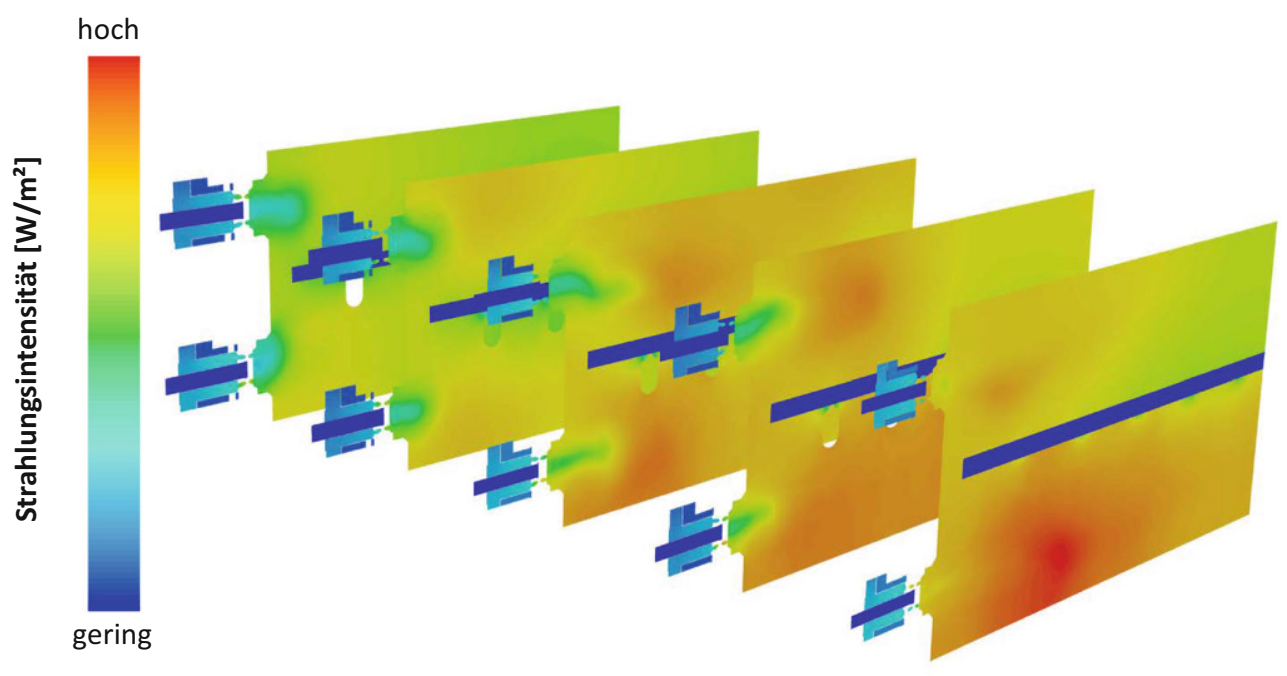


liche Leistungseinträge in die Bramme stattfinden. Durch Ändern des Ofenprogramms und alternativer Leistungseinstellungen an den Brennern konnten diese Effekte vermieden werden, was zu einer deutlich einheitlicheren Brammentemperatur am Ofenaustrag führt.

Funding. Open access funding provided by Montanuniversität Leoben.

Open Access Dieser Artikel wird unter der Creative Commons Namensnennung 4.0 International Lizenz (http://creativecommons.org/licenses/ by/4.0/deed.de) veröffentlicht, welche die Nutzung, Vervielfältigung,

Bearbeitung, Verbreitung und Wiedergabe in jeglichem Medium und Format erlaubt, sofern Sie den/die ursprünglichen Autor(en) und die Quelle ordnungsgemäß nennen, einen Link zur Creative Commons Lizenz beifügen und angeben, ob Änderungen vorgenommen wurden.

\section{Literatur}

1. Proceedings of the Third International Workshop on Measurement and Computation of Turbulent Nonpremixed Flames, 1998, S. 25-29 ULR: www.sandia.gov/TNF/images/inc/TNF3Proceedings. pdf.gz 03.05.2018.

2. Pfeiler, C.: CFD als Werkzeug in der Industrieofentechnik, BHM, 156 (2011), Nr. 9, 2011, S. 347-352

3. Peters, N.: Turbulent Combustion, Cambridge: University Press, 2000

4. Thynell, S. T.: Discrete-ordinates method in radiative heat transfer, International Journal of Engineering Science, 36 (1998), Nr. 12-14, S. 1651-1675

5. Barlow, R. S.; Karpetis, A. N.; Frank, J. H.; Chen, J.-Y.: Combust, Flame, 127 (2001), S. 2102-2118

6. Viscorova, R.: Untersuchung des Wärmeübergangs bei der Spritzwasserkühlung unter Berücksichtigung des Einflusses der Verzunderung, Dissertation, TU Clausthal, 2007 